Open Access

\title{
Robust power allocation for massive connections underlaying cognitive radio networks with channel uncertainty
}

\author{
Le Wang ${ }^{*}$, Guochun Ren, Jin Chen ${ }^{*}$, Guoru Ding, Zhen Xue and Haichao Wang
}

\begin{abstract}
This paper investigates a robust power allocation scheme for a cognitive radio network (CRN) with channel uncertainty, where a large number of secondary connections (SCs) share the same frequency spectrum with a primary user (PU). Specifically, considering the fact that the channel gain estimates from the secondary transmitters (S-Tx) to the primary receiver ( $\mathrm{P}-\mathrm{Rx}$ ) are typically uncertain and cannot be perfectly known in practice, this paper advocates a robust interference constraint. Meanwhile, we take the S-Tx's transmit power limitation and interference between SCs into account. The optimization problem is a non-convex and non-linear program (NCNLP) with an outage probability constraint. Both the original objective function and the outage probability constraint are converted into manageable forms via the mathematical transformation. Then, we introduce the convex optimization theory to solve the intractable problem. Additionally, we develop an efficient algorithm to obtain the near-optimal solution based on the interior point method, where we take advantage of the Newton algorithm to make the search of the feasible solution simple and effective. Finally, we conduct the in-depth simulations under various parameter configurations, which demonstrate that our proposed robust power allocation scheme can achieve higher performance in comparison to the existing scheme in the literature.
\end{abstract}

Keywords: Cognitive radio, Power allocation, Channel uncertainty, Convex optimization, Interference tolerability

\section{Introduction}

Since wireless personal communications have been in widespread applied to exchange information among individuals so far, not only are massive wireless devices growing up but also requirement of ubiquitous communications among individuals drives the development of abundant advanced wireless technologies [1]. In addition to this, the increasing demand for scarce frequency spectrum brings an exciting prospect to the development of the cognitive radio (CR) technology [2]. CR is a highly promising wireless technology which can mitigate the scarcity of spectral resources. In a cognitive radio network $(\mathrm{CRN})$, there is usually a primary user (PU) and massive secondary connections (SCs) sharing the same spectrum, i.e., they coexist with each other. There is a great deal of coexistence modes for a CRN. In general, we classify

*Correspondence: whcwl456@163.com; chenjin99@263.net College of Communications Engineering, PLA University of Science and Technology, Yudao Street, 210007 Nanjing, China the transmission modes into three types: overlay, interweave, and underlay modes. In the overlay mode, it is assumed that SCs have to acquire the perfect knowledge of the PU's messages. The secondary transmitters (S-Txs) send out their own signals along with the signal received from PU. The interference inflicted upon PU from SCs obtains the compensation via SC relaying the PU's signal. The interweave spectrum sharing mode is also called "opportunistic spectrum access" [3]. There is no cooperation between SCs and PU in the interweave mode. SC is allowed to occupy the channel if and only if the transmitter channels of SC and PU are mutually orthogonal. In time domain, SC should abandon the spectrum resource once the PU returned to utilize the channel occupied by SC. In the underlay mode, SCs are permitted to transmit concurrently with PU sharing the same spectrum bands, provided that the interference experienced by $\mathrm{PU}$ is controlled less than a certain threshold which PU can tolerate to [3]. 
This paper focuses on the underlay mode. It should be noted that the massive SCs will interfere with the communication of PU in general. A significant design challenge related to this network is how to maximize the throughput capacity of SCs with the quality of service (QoS) of the PU being guaranteed in the underlay mode. Moreover, when we consider the uncertainty of the channel between the $\mathrm{S}-\mathrm{Tx}$ and the primary receiver (P-Rx), this problem becomes more troublesome.

The optimal or suboptimal power allocation policies in the underlay mode have been already investigated widely [4-20]. A heuristic algorithm in CR systems under OFDMA has been reported in [4]. Ghasemi and Sousa [4] do not take the transmit power constraint of SCs into account. However, the transmit power constraint of SCs cannot be ignored due to the hardware of the S-Tx. In [5], ergodic and outage capacity of SCs over Rayleigh faded channel based on MMSE estimation is evaluated. The transmit power of the P-Rx is under the constraint of peak received power. In [6], transmission capacity of SCs is evaluated under the interference constraint of the PU. The SCs serve as cooperative relays in spectrum sharing environment. It is notable that perfect channel state information (CSI) is assumed in these works [4-11]. However, the CSI between SC and PU is usually difficult to be estimated accurately in actual situations mainly due to the lack of signaling cooperation between them. Therefore, power allocation with channel uncertainty should be considered in CRN [12-20]. A worst-case formulation is utilized in [12] for beamforming design, in which an ellipsoidal channel uncertainty region is assumed. Dall'Anese et al. [13] considers the probabilistic interference constraints for a CR power control problem, where uncertainty in composite fading channels comprising shadowing and Nakagami fading is accounted for. Power allocation problems for generic OFDMA systems with channel uncertainty have also been investigated extensively [14-16]. Ma et al. [17] shows the availability of imperfect CSI that includes probabilistic uncertainty and considers its worst effect when performing resource allocation. Different small cell networks is considered in [18], and there are tight interference constraints on PU. Power allocation along with relay selection schemes is optimized especially considering different channel uncertainty models in [18]. It is worth noting that [12-19] consider the channel uncertainty without the interference between massive SCs. Son et al. [20] study the power allocation problem via the water-filling algorithm. Furthermore, Son et al. [20] consider neither a great deal of SCs nor the interference constraints between SCs in the system. However, to the best of our knowledge, power allocation involving both channel uncertainty and mutual interference between massive SCs has not been addressed in the existing literature, which is the motivation of this work.
Differently, this paper investigates the problem of the robust power allocation for massive connections underlaying CRNs, where channel uncertainty and interference constraints are jointly considered. It is noted that the problem of interest is a non-convex and non-linear program (NCNLP), which is very difficult to solve. Specifically, to tackle with the practical problem inherent in power control with massive SCs, this paper makes the following contributions:

- Formulate an optimization problem which takes the SC's mutual interference constraints and channel uncertainty between the S-Tx and P-Rx into account, analyze the control of S-Tx's peak transmit-power constraints and adopt average interference to the probabilistic model for the QoS of the PU.

- Convert the original objective function and the outage probability constraint into a form which is easy to handle by some mathematical transformation and derive the analytical expressions via the convex optimization.

- Develop an efficient algorithm to obtain the near-optimal solution based on the interior point method under the strict interference constraint of the PU. Furthermore, the Newton algorithm is set for searching viable power value because it uses the second derivative information in the calculation of lowering direction and has a faster rate of decline.

- Conduct the in-depth simulations under various parameter configurations, such as outage probability and the number of SCs, which demonstrate that our proposed robust power allocation scheme can achieve higher performance in comparison to the state-of-the-art work in the literature.

The remainder of this paper is organized as follows. Section 2 presents our system model, and the optimization problem is formulated in Section 3, along with formulation analysis as well as problem transformation. The proposed power allocation algorithm is derived in Section 4. The results of extensive numerical simulations and the performance evaluation are presented in Section 5, followed by conclusions in Section 6 .

\section{System model}

Consider a CRN as shown in Fig. 1. There is a PU and massive SCs which utilize the same spectrum as the PU in the underlay mode. We denote $\mathcal{N}=\{1,2, \cdots, N\}$ as the set of all SCs. A connection consists of a S-Tx and a secondary receiver (S-Rx) and so does the PU. The SCs are supposed to be randomly distributed around the PU in the CRN. In addition, let $p_{\mathrm{PU}}$ and $p_{i}$ represent the transmit power of the PU and the $i$ th $\mathrm{SC}$, respectively. Also, let $p_{\max }$ denote the maximum transmit power of the S-Tx, $I_{\max }$ the maximum interference allowed by the $\mathrm{SC}$, and $I_{\max }^{p}$ 


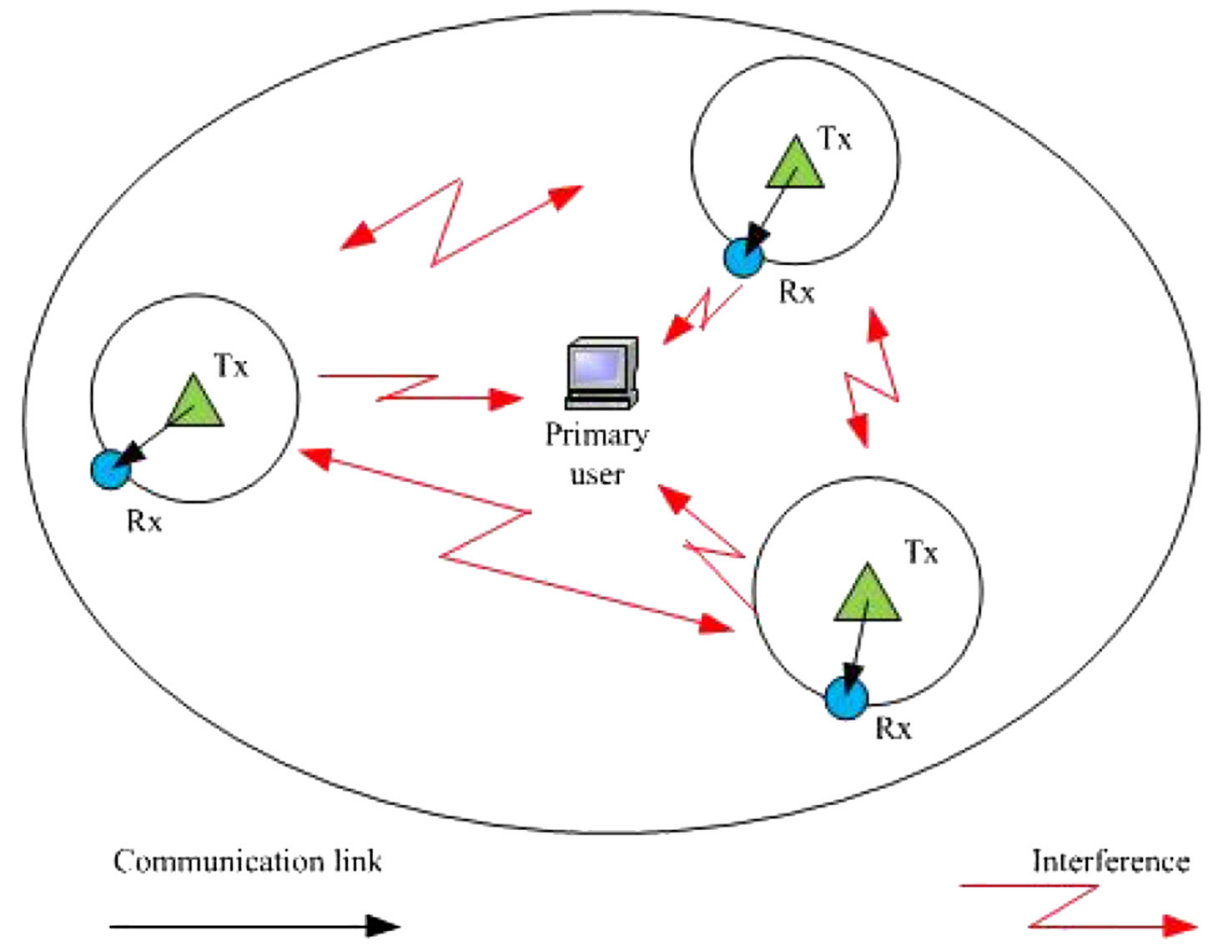

Fig. 1 A system model with the primary user and secondary connections sharing the same frequency spectrum

the maximum interference allowed by the PU. Moreover, vector $\mathbf{p}$ collects $\left\{p_{i}\right\}$.

The model of the channel propagation is made up of two main factors, i.e., large scale path loss and small scale multi-path fading. First and foremost, the power received by the receiver is described as follows:

$$
P_{\mathrm{R}}=P_{\mathrm{T}} * m_{r x, t x}^{-\beta} * h_{r x, t x}^{2}
$$

where $P_{\mathrm{R}}$ and $P_{\mathrm{T}}$ are the power of receiver and transmitter, respectively. $m_{r x, t x}$ is the large scale path loss from a transmitter to its receiver and $\beta$ is constant. $h_{r x, t x}$ is the small scale multi-path fading. Since we consider the spatial location distribution of SCs, so the large scale path loss is applied. Due to the multi-path effect, so we take the small scale multi-path fading into account. Then, we formulate the following expression to describe the large scale path loss from a transmitter to its receiver and it is the $\mathrm{dB}$ model of $m_{r x, t x}$ [21]:

$$
\begin{aligned}
M_{r x, t x}[\mathrm{~dB}] & =10 * \alpha \log (4 \pi d)+10 * \alpha \log (f) \\
& -20 \log \left(l_{T} * l_{R}\right),
\end{aligned}
$$

where $\alpha$ denotes the link loss coefficient which depends on the specific environment, $d$ is the distance in meters between the transmitter and the receiver, $f$ is the center frequency of transmitted signal and its unit is $\mathrm{MHz}, l_{\mathrm{T}}$ and $l_{\mathrm{R}}$ represent the height of the S-Tx and the S-Rx in meters, respectively. It is hard to establish the model of the small scale multi-path fading by rule and line. Generally speaking, we can obtain the similar effect qualitatively via certain probability distribution, such as Rayleigh distribution and Rice distribution [21]. It is assumed that the channel multi-path fading follows Rayleigh distribution, so it is modeled as

$$
h_{r x, t x}=\sqrt{h_{1}^{2}+h_{2}^{2}}
$$

where $h_{1}$ and $h_{2}$ are two independent Gaussian variables which follow the distribution of $N \sim\left(0, \delta^{2}\right)$.

The channel gain $g_{\mathrm{PU}} \geq 0$ between the primary transmitter (P-Tx) and P-Rx is assumed to have been easily acquired by some conventional channel estimation techniques [22]. Similarly, the channel gain $g_{i, j}$ between the $i$ th $\mathrm{S}-\mathrm{Tx}$ and the $j$ th $\mathrm{S}-\mathrm{Rx}$ is also known accurately, i.e., the STx has perfect channel state information [20]. Because SC and PU rarely have any cooperation during the process of their transmissions, the channel gain $g_{i}^{\mathrm{PU}}$ between them can be hardly estimated precisely unlike the channel gain between SCs.

As mentioned above, the signal-to-noise ratio (SNR) of the SC-Rx without any interference is

$$
\gamma_{i}=\frac{p_{i} g_{i, i}}{\sigma_{0}^{2}},
$$

where $\sigma_{0}^{2}$ is the power of the noise. Since we consider all the SCs sharing the same channel with the PU in the paper, 
hence, we can get the signal-to-interference-plus-noise ratio (SINR) of the S-Rx as follows:

$$
\mathrm{SINR}_{i}=\frac{p_{i} g_{i, i}}{\sum_{j=1, j \neq i}^{N} p_{j} g_{j, i}+\sigma_{0}^{2}},
$$

where $p_{i} g_{i, i}$ is the received signal power of the SC-Rx and $\sum_{j=1, j \neq i}^{N} p_{j} g_{j, i}$ represents the mutual interference of SCs. The network throughput capacity of all SCs is described as the following:

$$
T_{\text {sum }}=\sum_{i=1}^{N} \log _{2}\left(1+\mathrm{SINR}_{i}\right) .
$$

As to the constraints, there are basically three types on the power allocation. One of them is that any transmit power of the S-Tx is limited to the power budget [20]:

$$
p_{i} \leq p_{\max }, \forall i \text {. }
$$

Due to the mutual interference, the secondary ones are the interference constraints with respect to the SCs, which are formulated as follows:

$$
\sum_{i=1, j \neq i}^{N} p_{i} g_{i, j}=I^{(-n)} \leq I_{\max }, \forall i .
$$

The final constraint concentrates on the protection for the PU. We assume that the PU has an average interference power tolerable capability. In other words, the SCs can coexist with the $\mathrm{PU}$ as long as the average interference power from all the S-Txs is limited below than a certain threshold [20]. It is necessary to explain the "average". Because the interference from SCs is time-varying, we cannot guarantee that there will be always weak interference. If so, it is likely that there is some strong interference from several certain S-Txs. Thus, the weak one can make compensation for the PU's performance in average. We define $\operatorname{Pr}[$.$] as the outage probability that the interference$ to the PU is more than a threshold value. We can write it as the following expression:

$$
\operatorname{Pr}\left[\sum_{i=1}^{N} p_{i} g_{i}^{P U} \leq I_{\max }^{p}\right] \geq 1-\varepsilon,
$$

where the channel gain $g_{i}^{\mathrm{PU}}$ is an independent and identically distributed exponential random variable with mean $\theta$ and $\varepsilon$ is the outage probability.

Besides, in this CRN system, there is the interference from the P-Tx to S-Rx. Set $g_{\mathrm{PU}}^{i}$ as the channel gain between the P-Tx and S-Rx, which is perfectly known. Figure 2 reflects the system performance including the interference from the P-Tx to S-Rx. In the scenario of this paper, we assume that the P-Tx is far away from SCs and there is massive mutual interference between massive SCs which is much more than the interference from PTx. Based on the above phenomenon and explanations, we

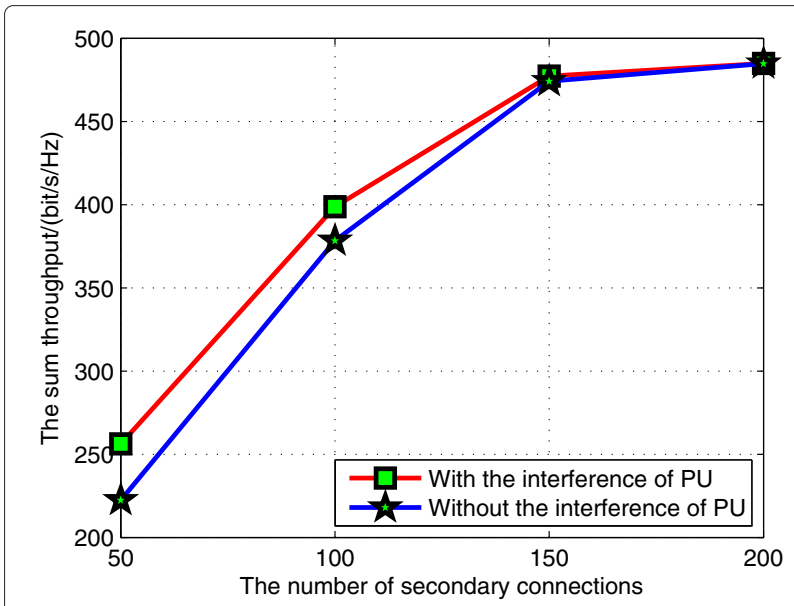

Fig. 2 The system performance including the interference from the P-Tx to S-Rx in comparison with that excluding it

ignore the interference from the P-Tx when focusing on the optimization of the SCs' capacity.

As we noted above, the optimization objective is to maximize the capacity of the SCs via determining the allocation of the optimal transmit power vector $\mathbf{p}=$ $\left\{p_{1}, p_{2}, p_{3}, \cdots p_{N}\right\}$ of S-Txs , namely:

$$
\begin{array}{ll}
(\mathcal{P}) & \max _{\mathbf{p}=\left\{p_{i}\right\}} T_{\text {sum }} \\
\text { s.t. } & C 1: p_{i} \leq p_{\max }, \forall i \\
& C 2: \sum_{j=1, j \neq i}^{N} p_{j} g_{j, i}=I^{(-n)} \leq I_{\max }, \forall i \\
& C 3: \operatorname{Pr}\left[\sum_{i=1}^{N} p_{i} g_{i}^{P U} \leq I_{\max }^{p}\right] \geq 1-\varepsilon .
\end{array}
$$

In (10), the goal of the optimal problem $(\mathcal{P})$ is to find the optimal power allocation method to maximize the total throughput capacity of the system. For the sake of the description convenience, we regard $(\mathcal{P})$ as the original problem. Among the three constraints, the first one is to restrict the transmit power of S-Tx, the second one ensures the coexistence of the communication between the SCs, and the final one focuses on the protection for the PU. However, since the objective function is non-linear and it is not even convex, it will become very complicated if we directly solve the optimization problem of the above form. Additionally, the mutual influence actually exists between multiple variables. Therefore, not only does the problem become tricky but also the complexity will rise steeply as the number of the variables increases. Paper [23, 24 ] suggest that a large number of iterations is needed in order to tackle with this problem. Because of the structure of the function, this problem cannot directly use the methods proposed by $[25,26]$. As a result, intractability 
and unavailability of the uncertain channel gain along with SCs' interference make the non-linear and non-convex expression mathematically difficult to handle. In addition, there is not yet any satisfactory solution to the open issue so far. In the following part, we try to introduce a feasible way to tackle with the difficulty, which takes advantage of the convex optimization theory.

\section{Problem transformation}

We can see that the problem (10) is an optimization problem with some constraints. The convex optimization is a powerful tool to solve these kinds of problems. A standard form of convex optimization problems is formulated as follows:

$$
\begin{array}{cl}
\min & f_{0}(x) \\
\text { s.t. } & f_{i}(x) \leq 0, i=1, \cdots, q \\
& a_{j}^{T} x=b_{j}, j=1, \cdots, p
\end{array}
$$

where all of $f_{0}, \cdots, f_{q}$ are convex functions, $f_{i}(x)$ is the constraint of inequality, and $h_{j}(x)=a_{j}^{T} x-b_{j}$ is the constraint of equality [27]. But as it is shown in problem (10), neither optimization objective nor the constraints is convex function. The convex optimization cannot be used straightly. So, we first investigate the primal objective function and approximate it with a series of convex ones.

The objective function can be rewritten as the difference of two concave functions, $u$ and $w$ :

$$
\sum_{i=1}^{N} \log _{2}\left(1+\frac{p_{i} g_{i, i}}{\sum_{j=1, j \neq i}^{N} p_{j} g_{j, i}+\sigma_{0}^{2}}\right)=u(\mathbf{p})-w(\mathbf{p}),
$$

where both $u(\mathbf{p})$ and $w(\mathbf{p})$ are concave functions:

$$
\begin{aligned}
& u(\mathbf{p})=\sum_{i=1}^{N} \log _{2}\left(\sum_{j=1}^{N} p_{j} g_{j, i}+\sigma_{0}^{2}\right) \\
& w(\mathbf{p})=\sum_{i=1}^{N} \log _{2}\left(\sum_{j \neq i}^{N} p_{j} g_{j, i}+\sigma_{0}^{2}\right) .
\end{aligned}
$$

Then, we work out the derivation of $w(\mathbf{p})$ about $p_{i}$ :

$$
\begin{aligned}
\frac{\partial w(\mathbf{p})}{\partial p_{i}} & =\sum_{j \neq i}^{N} \frac{1}{\ln 2} \frac{g_{j, i}}{\sum_{j \neq i}^{N} p_{j} g_{j, i}+\sigma_{0}^{2}} \\
& =\sum_{j=1}^{N} \frac{1}{\sum_{j \neq i}^{N} p_{j} g_{j, i}+\sigma_{0}^{2}} e_{j, i},
\end{aligned}
$$

where $e_{j, i}=\left\{\begin{array}{l}0, j=i \\ \frac{g_{j, i}}{\ln 2}, j \neq i\end{array}\right.$. The equation above is represented as a column vector $\nabla w(\mathbf{p})=\left\{\tau_{i}\right\}=\left\{\frac{\partial w(\mathbf{p})}{\partial p_{i}}\right\}$. We can approximately denote $w(\mathbf{p})$ by the first-order Taylor series expansion $w\left(\mathbf{p}^{(k)}\right)+\left\langle\nabla w\left(\mathbf{p}^{(k)}\right), \mathbf{p}-\mathbf{p}^{(k)}\right\rangle$ of $w(\mathbf{p})$ around the given any viable solution $\left\{p_{i}^{k}\right\}$. It is important to note that the superscript $k$ represents the number of iterations. As a result, a conservative surrogate for the objective function is formulated as follows:

$$
-f_{0}(\mathbf{p})=u(\mathbf{p})-w\left(\mathbf{p}^{(k)}\right)-\left\langle\nabla w\left(\mathbf{p}^{(k)}\right), \mathbf{p}-\mathbf{p}^{(k)}\right\rangle .
$$

Since $w(\mathbf{p})$ is concave function as mentioned previously, it can be easily captured as follows:

$$
w(\mathbf{p}) \leq w\left(\mathbf{p}^{(k)}\right)+\left\langle\nabla w\left(\mathbf{p}^{(k)}\right), \mathbf{p}-\mathbf{p}^{(k)}\right\rangle .
$$

If $\left\{p_{i}\right\}$ is feasible and $-f_{0}\left(\mathbf{p}^{(k+1)}\right) \geq-f_{0}\left(\mathbf{p}^{(k)}\right)$, the following formulation can be acquired:

$$
u\left(\mathbf{p}^{(k+1)}\right)-w\left(\mathbf{p}^{(k+1)}\right) \geq-f_{0}\left(\mathbf{p}^{(k+1)}\right) \geq u\left(\mathbf{p}^{(k)}\right)-w\left(\mathbf{p}^{(k)}\right) .
$$

It is can be seen that $-f_{0}\left(\mathbf{p}^{(k)}\right)$ provides a lower bound. Maximizing function (12) is equivalent to maximizing the initial function. Through the above analysis, the original problem is non-convex, but we can approximate it with convex functions.

Here, we analyze the characteristics of the constraints. Considering the fact that CSI can be robustly estimated between SCs, $C 1$ is a simple linear constraint, so is $C 2$. They can be equivalently written as:

$$
\begin{aligned}
& f_{1, i}(\mathbf{p})=p_{i}-p_{\max } \leq 0, \forall i \\
& f_{2, i}(\mathbf{p})=\sum_{j=1, j \neq i}^{N} p_{j} g_{j, i}-I_{\max } \leq 0, \forall i .
\end{aligned}
$$

Nevertheless, for constraint $C 3$, the uncertainty of the channel between S-Tx and P-Rx makes C3 intractable, i.e., $C 3$ has no idea to satisfy the requirement of a convex function. As a result, we take different attitudes towards the three constraints. For the first two, we can acquire the goal through above steps (17). As to $C 3$, we have to study its transformation as the following description.

Let $X_{i}=p_{i} g_{i}^{\mathrm{PU}}$, which are independently exponential distributed with mean $p_{i} \theta$. Denote $X=\sum_{i} X_{i}$ as the sum of all the random variables. Therefore, we can change the original constraint into the following probability form:

$$
\operatorname{Pr}\left[X=\sum_{i} X_{i} \leq I_{\max }^{p}\right] \geq 1-\varepsilon
$$

How to test and verify the constraint (19)? The key lies in the distribution of $X$. To determine the distribution explicitly, we use Gaussian approximation based on the following Lemma 1 [28].

Lemma 1. The Lyapunov's central limit theorem (CLT): If $X_{1}, X_{2}, \ldots, X_{n}$ are independent of each other with mean 
$E\left(X_{k}\right)=\mu_{k}$ and variance $D\left(X_{k}\right)=\sigma_{k}^{2}>0$,we can obtain that

$$
Z_{n}=\frac{\sum_{k=1}^{n} X_{k}-\sum_{k=1}^{n} \mu_{k}}{B_{n}} \sim N(0,1)
$$

and

$$
\sum_{k=1}^{n} X_{k} \sim N\left(\sum_{k=1}^{n} \mu_{k}, B_{n}^{2}\right)
$$

where $B_{n}^{2}=\sum_{k=1}^{n} \sigma_{k}^{2}$

Due to the above Lemma 1, the Lyapunov condition should be satisfied as follows to apply the Lyapunov's (CLT):

$$
\lim _{N \rightarrow \infty} \frac{\left(\sum_{i=1}^{N} r_{i}^{3}\right)^{\frac{1}{3}}}{\left(\sum_{i=1}^{N} \sigma_{i}^{3}\right)^{\frac{1}{2}}}=0 .
$$

where $r_{i}$ is the third-order center distance of $X_{i}$, i.e., $E\left[\left(X_{i}-m_{i}\right)^{3}\right]$ and $m_{i}$ and $\sigma_{i}^{2}$ are the mean and variance of the exponential distributed random variable $X_{i}$, respectively. We can easily check this condition [20].

In general, for massive connections, we can regard $X$ as a normally distributed random variable whose mean is $m$ and variance is $\sigma^{2}$ approximately

$$
\begin{aligned}
& m \sim \sum_{i} p_{i} \lambda \\
& \sigma^{2} \sim \sum_{i}\left(p_{i} \lambda\right)^{2} .
\end{aligned}
$$

Therefore, the following constraint is a conservative surrogate for (19):

$$
\begin{aligned}
P(\mathbf{p}) & =1-F_{N}\left(I_{\max }^{p}\right) \\
& =\frac{1}{2} \operatorname{erfc}\left(\frac{I_{\max }^{p}-m}{\sqrt{2} \sigma}\right) \leq \varepsilon .
\end{aligned}
$$

where $F_{N}(\cdot)$ is the cumulative distribution function (CDF) of a normal distribution with mean $m$ and variance $\sigma^{2}$ and $\operatorname{erfc}(z)=\frac{2}{\sqrt{\pi}} \int_{z}^{\infty} e^{-t^{2}} d t$. For (24), we assume that $f_{3}(\mathbf{p})=\frac{1}{2} \operatorname{erfc}\left(\frac{I_{\max }^{p}-m}{\sqrt{2} \sigma}\right)-\varepsilon$. It is obvious that it is not a standard convex function at all which needs separate treatment. Inspired by the scheme proposed in [20], once a power allocation is given from the first two constraints, we can check whether it satisfies the outage constraint (24) or not.

In a word, we can easily confirm that $(\mathcal{P})$ is equivalent to

$$
\begin{array}{ll}
(\mathcal{P} 1) & \min f_{0}(\mathbf{p}) \\
\text { s.t. } & C 1: f_{1, i}(\mathbf{p})=p_{i}-p_{\max } \leq 0, \quad \forall i \\
& C 2: f_{2, i}(\mathbf{p})=\sum_{j=1, j \neq i}^{N} p_{j} g_{j, i}-I_{\max } \leq 0, \quad \forall i \\
& C 3: f_{3}(\mathbf{p})=\frac{1}{2} \operatorname{erfc}\left(\frac{I_{\max }^{p}-m}{\sqrt{2} \sigma}\right)-\varepsilon \leq 0 .
\end{array}
$$

Since $C 3$ is not a standard convex function at all as abovementioned, it does not satisfy the standard form of convex optimization problems (11). So we could not deal with the problem $(\mathcal{P} 1)$ directly. In order to obtain a feasible solution, we divide the original problem $(\mathcal{P} 1)$ into two parts to solve in this paper:

$$
\begin{array}{r}
(\mathcal{S P} 1): \min f_{0}(\mathbf{p}) \\
\text { s.t. } C 1, C 2
\end{array}
$$

and

$$
(\mathcal{S P} 2): f_{3}(\mathbf{p})=\frac{1}{2} \operatorname{erfc}\left(\frac{I_{\max }^{p}-m}{\sqrt{2} \sigma}\right)-\varepsilon \leq 0 .
$$

If we acquire an optimal solution $\mathbf{p}$ from $(\mathcal{S P} 1)$, we will examine whether it meets or not by substituting into $(\mathcal{S P} 2)$. In another word, the input of $(\mathcal{S P} 2)$ is the output of $(\mathcal{S P} 1)$.

For the first part $(\mathcal{S P} 1)$, it is suitable to use the above convex optimization to deal with. The Lagrangian function of the problem $(\mathcal{S P} 1)$ is given by

$$
\begin{aligned}
L(\mathbf{p}, \lambda, v) & =f_{0}(\mathbf{p})+\sum_{i=1}^{N} \lambda_{i}\left(p_{i}-p_{\max }\right) \\
& +\sum_{i=1}^{N} v_{i}\left(\sum_{j=1, j \neq i}^{N} p_{j} g_{j, i}-I_{\max }\right),
\end{aligned}
$$

where $\lambda$ and $v$ are the non-negative dual variables associated with the corresponding constraints $C 1$ and $C 2$, respectively. The Lagrangian dual function is defined as follows:

$$
\begin{aligned}
G(\lambda, v)= & \inf _{\mathbf{p}=\left\{p_{i}\right\}} L(\mathbf{p}, \lambda, v) \\
= & \inf _{\mathbf{p}=\left\{p_{i}\right\}}\left(f_{0}(\mathbf{p})+\sum_{i=1}^{N} \lambda_{i}\left(p_{i}-p_{\max }\right)\right. \\
& \left.+\sum_{i=1}^{N} v_{i}\left(\sum_{j=1, j \neq i}^{N} p_{j} g_{j, i}-I_{\max }\right)\right) .
\end{aligned}
$$

If there is no lower bound of the Lagrangian, the dual function value is $-\infty$.

Next, we carry on the feasibility analysis of the part. Problem (26) is feasible if and only if the following linear programming (LP) is feasible: 
$\left(\mathcal{S P} 1^{\prime}\right)$ : find $\mathbf{p}$

$$
\begin{aligned}
\text { s.t. } C 1: f_{1}(\mathbf{p}) & =p_{i}-p_{\max } \leq 0, \forall i \\
C 2: f_{2}(\mathbf{p}) & =\sum_{j=1, j \neq i}^{N} p_{j} g_{j, i}-I_{\max } \leq 0, \quad \forall i .
\end{aligned}
$$

The corresponding dual function is given by

$$
\begin{aligned}
L^{\prime}\left(\mathbf{p}, \lambda^{\prime}, v^{\prime}\right) & =\sum_{i=1}^{N} \lambda^{\prime}{ }_{i}\left(p_{i}-p_{\max }\right) \\
& +\sum_{i=1}^{N} v^{\prime}{ }_{i}\left(\sum_{j=1, j \neq i}^{N} p_{j} g_{j, i}-I_{\max }\right),
\end{aligned}
$$

where $\lambda^{\prime}=\left[\lambda^{\prime}{ }_{1}, \cdots, \lambda^{\prime}{ }_{N}\right] \geq 0$ and $v^{\prime}=\left[v^{\prime}{ }_{1}, \cdots, v_{N}^{\prime}\right] \geq$ 0 consist of the Lagrange multipliers associated with the $N$ connections constraints. The dual function of problem $\left(S P 1^{\prime}\right)$ is then given by

$$
G^{\prime}\left(\lambda^{\prime}, v^{\prime}\right)=\inf _{\mathbf{p}=\left\{p_{i}\right\}} L^{\prime}\left(\mathbf{p}, \lambda^{\prime}, v^{\prime}\right) .
$$

Theorem 1. For a given $I_{\max }>0, p_{\max }>0$, problem $\left(\mathcal{S P} 1^{\prime}\right)$ is infeasible if and only if there exists $\lambda^{\prime} \geq 0, v^{\prime} \geq 0$ such that $G^{\prime}\left(\lambda^{\prime}, v^{\prime}\right)>0$; otherwise, $\left(\mathcal{S P} 1^{\prime}\right)$ is feasible.

Proof. See Appendix for details.

For the second part $(\mathcal{S P} 2)$, we can check whether the optimal value $p t^{*}$ obtained by $(\mathcal{S P} 1)$ satisfies the outage constraint (27) or not. If it does, we think it is the optimal solution to $\mathcal{P} 1$; otherwise, all the transmit power decreases by a step $\gamma$ and we search for the optimal one again.

Since the original complex problem is transformed into a more tractable form, we obtain the near-optimal solution along with acceptable computational complexity. Detailed algorithm design will be provided in the following section.

\section{Power allocation algorithm}

In the above section, we transfer the constraint $C 3$ into a tractable form and approximate the original problem with convex functions. As is mentioned, once a power allocation is given, we can check whether it satisfies the outage constraint $C 3$ or not. So our power allocation algorithm consists of two steps. Firstly, we apply the convex optimization to getting over the problem $(\mathcal{S P} 1)$ based on the interior method. Note that the optimal solution must satisfy the constraints $C 1$ and $C 2$. Then, we should check the optimal solution whether satisfies the outage constraint $C 3$ or not. If it does, the optimal solution of problem $(S \mathcal{P} 1)$ is also the optimal solution of the original problem; otherwise, all the transmit power decreases by a step $\gamma$ until the constraint $C 3$ is satisfied.
The duality properties should be analyzed first of all, which can demonstrate the existence of the optimum solution. We formulate the optimal value of the Lagrange dual problem as follows:

$$
d^{*}=\underset{\lambda, v \geq 0}{\operatorname{maximize}} G(\lambda, v) .
$$

It is assumed that $o p t^{*}$ is the optimal value of the problem $(\mathcal{S P} 1)$. We denote the optimal duality gap of the primal problem by the difference $o p t^{*}-d^{*}$. If the strong duality is established, the optimal duality gap will hold zero [29]. In particular, $(\mathcal{S P} 1)$ is convex and satisfies Slater's condition [27]: there exists any valid $\mathbf{p}$ and this can make the following expressions hold,

$$
\begin{aligned}
& p_{i}<p_{\max }, \forall i \\
& \sum_{j=1, j \neq i}^{N} p_{j} g_{j, i}<I_{\max }, \forall i,
\end{aligned}
$$

where the inequality constraints must meet the condition strictly. Since $(\mathcal{S P} 1)$ is convex as is mentioned above and we can learn from Slater's theorem that the Lagrangian dual function (29) satisfies the strong duality, so it also satisfies the following important theorem [27] which is its sufficient and necessary condition.

Lemma 2. If both an objective function and its constraints of a convex optimization problem are differentiable and satisfy Slater's condition, we can capture that the Karush-Kuhn-Tucker (KKT) [27] conditions are the sufficient and necessary conditions for obtaining the optimality of the solution:

$$
\begin{aligned}
\nabla f_{0}\left(\mathbf{p}^{*}\right) & +\nabla\left(\sum_{i=1}^{N} \lambda_{i}^{*}\left(p_{i}^{*}-p_{\max }\right)\right) \\
& +\nabla \sum_{i=1}^{N} v_{i}^{*}\left(\sum_{j=1, j \neq i}^{N} p_{j}^{*} g_{j, i}-I_{\max }\right)=0 .
\end{aligned}
$$

The above formulation is only one of the KKT conditions. According to the Lemma 2 mentioned above, we can ensure that the optimal duality gap is zero. So when $\mathbf{p}^{*}$ is optimal, we can attain the dual optimum as well as $\lambda^{*}, v^{*}$. Nevertheless, we can hardly tackle with the KKT conditions analytically of the intractable problem in this paper. As a result, we introduce the interior point method to deal with the problem together with the KKT conditions.

It can be seen that the non-convex optimization problem $(\mathcal{P})$ has already been approximated by $(\mathcal{P} 1)$ in which only the third constraint is not convex. Then, we research for the existence of an optimal solution. Moreover, the optimality conditions (KKT conditions) are captured. In this section, we attempt to develop a quick and effective iterative algorithm. The barrier method is introduced to 
search for the global optimal solution throughout the previous analysis. As is well known, the barrier method is a representative technique of the class of interior point methods. It usually contains a logarithmic barrier function and the Newton method for minimization without constraints [21]. The advantage of the barrier method is that it is simple to implement and it can surely outperform other methods in performance.

Newton algorithm is a universal descent method, where the iteration consists of damping Newton stage and quadratic convergence stage, i.e., pure Newton stage. The trail of steps is the direction of searching in Newton algorithm. The backtracking line search is applied to the iteration, which is a kind of non-accurate line search approach. Newton algorithm plays a role as the internal iteration of the interior point method. In terms of searching the feasible solution, it works simply and effectively [27].

Next, we have formulated the following approximation of the inequality:

$$
\min f_{0}(\mathbf{p})+\frac{1}{t} \phi(\mathbf{p}), t>0,
$$

where $\phi(\mathbf{p})=-\sum_{i=1}^{N} \log \left(-f_{1, i}(\mathbf{p})-f_{2, i}(\mathbf{p})\right)$ is called the logarithmic barrier function of problem $(\mathcal{S P} 1)$. Because $-(1 / t) \log (-u)$ is convex, differentiable, and increasing when $u<0$, Newton algorithm can be applied to searching for the minimum of (35) within $p_{i} \in\left[0, p_{\text {max }}\right]$. Why do we select Newton algorithm? As is mentioned above, it uses the second derivation information when computing the descent direction. It exhibits the quite quick convergence rates. As [29] suggests, the approximation quality will improve apparently as the parameter $t$ grows.

Finally, the overall procedure for solving the optimization problem is presented in the following algorithm.

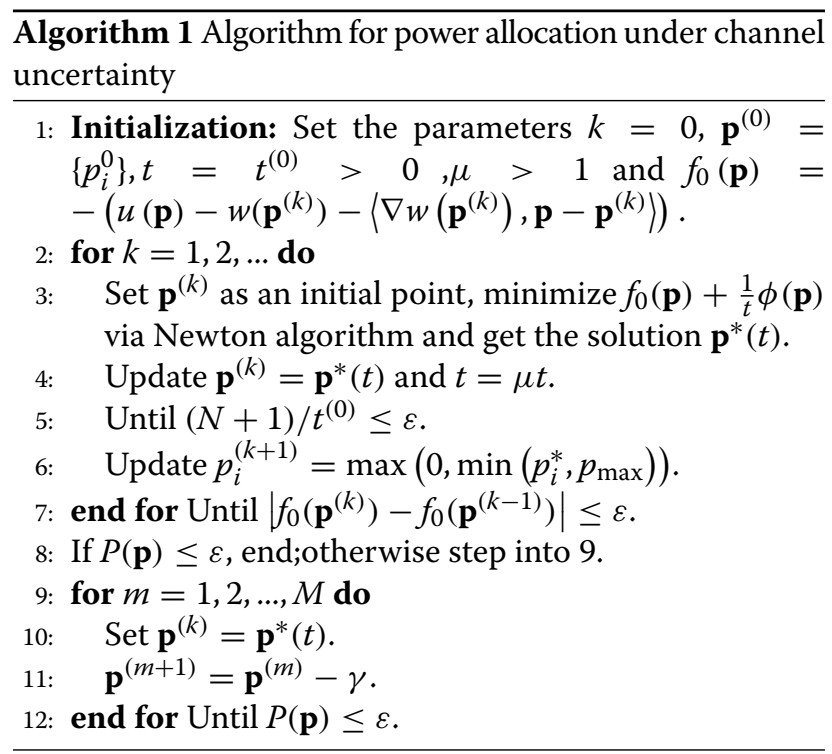

In order to verify its effectiveness, the proposed algorithm is compared with the algorithm in paper [20] and this will be discussed further with the simulation results.

\section{Performance evaluation}

The proposed power allocation algorithm is verified via the following numerical tests. In Table 1, we list the system parameters used in the simulations.

The presented results are obtained by simulating 1000 independent trials and then taking the expected value. To benchmark performance of the proposed near-optimal scheme, a simple suboptimal algorithm in paper [20] is considered. In paper [20], Son et al. investigate power allocation algorithms for OFDM-based cognitive radio systems, where the intra-system channel state information (CSI) of the SC is perfectly known and the inter-system CSI is only partially available to the S-Tx. They develop a suboptimal power allocation algorithm, which repeatedly solves a sub-problem having only a transmit-power constraint and then adjusts the available transmit power until the desired outage probability is achieved. In this paper, we regard this algorithm as a comparison with our proposed algorithm. More information can be referred to paper [20].

Figure 3 illustrates the simulation scenario for the sum throughput under the different algorithms where $\gamma=$ $0.2, \varepsilon=0.05$. It is assumed that there are 50 SCs. We can see that the proposed algorithm can acquire higher throughput than the algorithm proposed in [20]. When each S-Tx uses the maximum transmit power, the sum throughput is highest, but the strong interference on the PU limits the transmit power. On the other hand, from the tendency of the curve in Fig. 3, it can be observed that the sum throughput derived from our proposed scheme has always outperformed the other one derived from paper [20]. This is due to the algorithm proposed in [20], which is based on the binary search, causes great waste of power resource. Hence, the proposed algorithm can perform better. In our paper, when the number of SUs becomes large, the corresponding iteration will be more. Additionally, $-f_{0}(\mathbf{p})$ as the approximate lower bound of the best solution updates itself after each iteration. In this case, $-f_{0}(\mathbf{p})$ will become more and more close to the optimal solution.

Table 1 System parameters used in simulations

\begin{tabular}{lll}
\hline Parameter & Value & Comments \\
\hline$f$ & $700 \mathrm{MHz}$ & Center frequency of transmitted signal \\
$\sigma_{0}^{2}$ & $-130 \mathrm{dBm}$ & Noise power \\
$\alpha$ & 4 & Path loss exponent \\
$I_{T}, I_{R}$ & $2 \mathrm{~m}$ & Antenna height of the S-Tx, S-Rx \\
$P_{\max }$ & $25 \mathrm{dBm}$ & The maximum power of the S-Tx \\
$I_{\max }, I_{\max }^{p}$ & $-120 \mathrm{dBm}$ & The interference threshold value of SC,PU
\end{tabular}




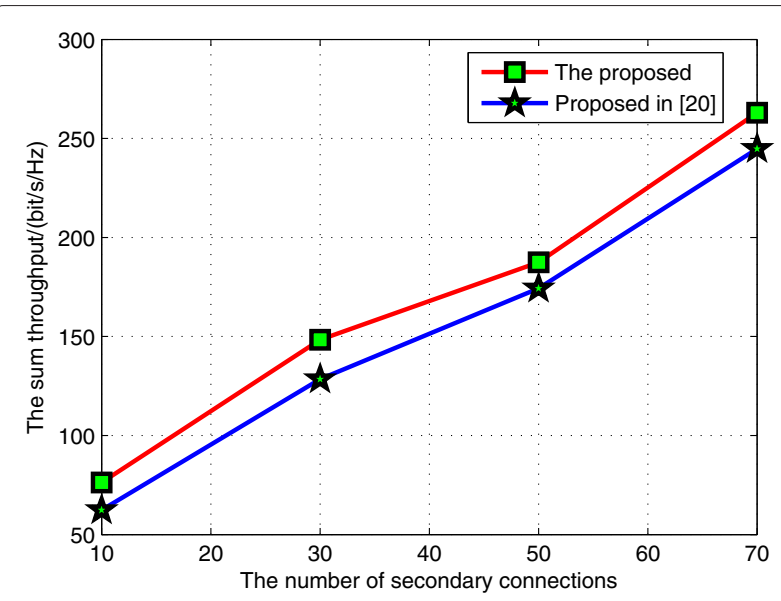

Fig. 3 The sum throughput comparison of the proposed and suboptimal algorithms

Therefore, our proposed algorithm is able to obtain the better effect of the system performance.

Figure 4 compares the spectral efficiency in the cases of different step values, where the number of SCs is assumed 50. A few interesting observations can be obtained. It is shown that the rapidity of the convergence correlates closely with the value of $\gamma$. That is to say, the convergence of the sum throughput turns faster as $\gamma$ becomes larger. Moreover, the faster the convergence is, the lower the accuracy is and the deviation increases when the accuracy of the transmit power becomes lower. Therefore, the value of $\gamma$ is better to the optimal transmit-power if it is smaller. Notably, $\gamma$ should be set within $[0.05,0.25]$ through a lot of tests and trials. In Fig. 5, $\gamma$ is set as 0.1. We examine the sum throughput by varying the outage probability $\varepsilon$. The number of SCs still remains 50 . There are some conclusions that can be drawn from Fig. 5 including that the throughput improves as $\varepsilon$ grows larger, and the outage

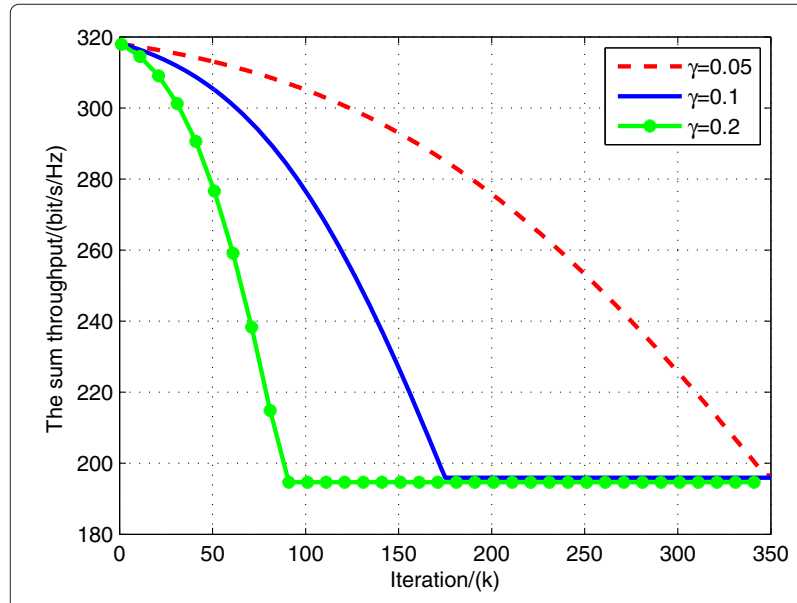

Fig. 4 Performance comparison of different values of $\gamma$

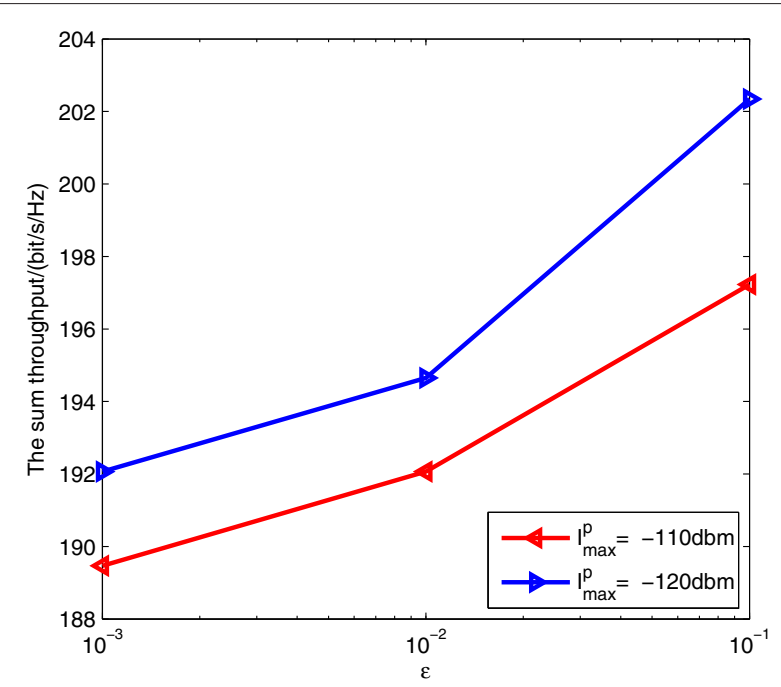

Fig. 5 The sum throughput versus $\varepsilon$

probability and the interference threshold have the same impact on the sum throughput.

Figure 6 discloses the average throughput of each SC and the sum throughput in terms of different numbers of SCs. In this figure, the left vertical axis represents the average throughput of each $\mathrm{SC}$ and the right one is the sum throughput. As can be seen obviously, with the number of SCs increasing, the average throughput is decreasing and the sum throughput rises first and then gradually falls. When there are about $160 \mathrm{SCs}$, the up trend of the sum throughput becomes gentle since the interference constraints between SCs has offset the rise in throughput. When there are about $210 \mathrm{SCs}$, the sum throughput declines sharply. This is because when the number of SCs increases seriously, the interference between SCs is also increasing seriously, even more than the throughput. It is

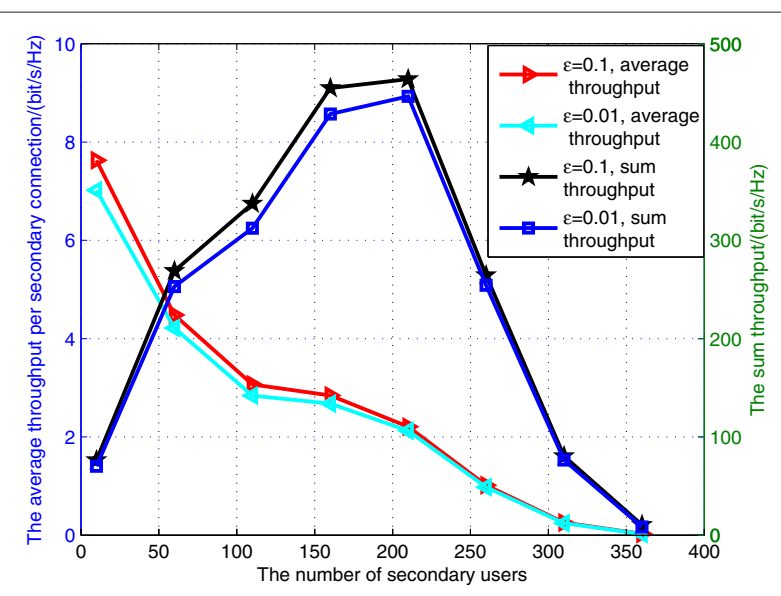

Fig. 6 Performance comparison under different values of $\varepsilon$ 
also observed that both of the average throughput of each SC and the sum throughput under $\varepsilon=0.01$ are extremely close to those under $\varepsilon=0.2$. In a word, the constraint of the PU becomes more and more relaxed with the number of the users increasing. On the one hand, if the number of SCs is large, the interference constraints of SCs play a dominant role in the problem. On the other hand, when there are a small number of SCs, however, the interference limitation of PU would become principal.

\section{Conclusions}

In this paper, the robust power allocation scheme solved the problem on how to maximize the throughput capacity of SCs on the condition that the quality of service of the PU would be ensured. This problem was formulated for a CRN with massive connections. Due to the uncertainty present in the channel between the S-Tx and the $\mathrm{P}-\mathrm{Rx}$, a robust interference constraint with probability was imposed to protect the PU system, which utilized the convex optimization theory. In this paper, we also introduced the iterated algorithm including Newton algorithm based on the interior point method and compared it with the algorithm proposed in [20]. We evaluated the spectral efficiency through extensive simulations and showed that the SCs can achieve higher performance in the proposed algorithm than that in the algorithm showed in [20]. As future work, a subject of extension to more general channel models including correlation or feedback delay might be researched further.

\section{Appendix}

\section{The proof of Theorem 1}

We first prove the "if" part. If $\mathbf{p}$ is a feasible solution given $I_{\max }>0, p_{\max }>0$, then for any $\lambda^{\prime} \geq 0, v^{\prime} \geq 0$ it follows that

$$
G^{\prime}\left(\lambda^{\prime}, v^{\prime}\right) \leq L^{\prime}\left(\mathbf{p}, \lambda^{\prime}, v^{\prime}\right) \leq 0
$$

and thus $\max _{\lambda, v} G^{\prime}\left(\lambda^{\prime}, v^{\prime}\right) \leq 0$, which contradicts with the given assumption that there exists an $\lambda^{\prime} \geq 0, v^{\prime} \geq 0$ such that $G^{\prime}\left(\lambda^{\prime}, v^{\prime}\right)>0$. The part is thus proved.

Then, we prove the "only if" part of Theorem 1 by showing that its transposition is true. Suppose that problem $\left(\mathcal{S P} 1^{\prime}\right)$ is feasible and there exists $\lambda^{\prime} \geq 0, v^{\prime} \geq 0$ such that $G^{\prime}\left(\lambda^{\prime}, v^{\prime}\right)>0$. However, since $\left(\mathcal{S P} 1^{\prime}\right)$ is assumed to be feasible and $\lambda^{\prime} \geq 0, v^{\prime} \geq 0$, there exists a solution such that it can satisfy all the constraints:

$$
\begin{array}{r}
\lambda_{i}{ }^{\prime}\left(p_{i}-p_{\max }\right) \leq 0 \quad \forall i \in N \\
v_{i}{ }^{\prime}\left(\sum_{j=1, j \neq i}^{N} p_{j} g_{j, i}-I_{\max }\right) \leq 0 \quad \forall i \in N .
\end{array}
$$

We thus have $G^{\prime}\left(\lambda^{\prime}, v^{\prime}\right) \leq L^{\prime}\left(\mathbf{p}, \lambda^{\prime}, v^{\prime}\right) \leq 0$.
This contradicts $G^{\prime}\left(\lambda^{\prime}, v^{\prime}\right)>0$, and thus, problem $\left(\mathcal{S P} 1^{\prime}\right)$ is feasible if $G^{\prime}\left(\lambda^{\prime}, v^{\prime}\right)<0$. The "only if" part is thus proved.

Combining the above proofs of both "if" and "only if" parts, Theorem 1 thus follows.

\section{Competing interests}

The authors declare that they have no competing interests.

\section{Authors' information}

Guoru Ding is with National Mobile Communications Research Laboratory, Southeast University, Nanjing 210096, China, and also with College of Communications Engineering, PLA University of Science and Technology, Yudao Street, 210007 Nanjing, China. The other authors are with College of Communications Engineering, PLA University of Science and Technology, Yudao Street, 210007 Nanjing, China.

\section{Acknowledgements}

This work is supported in part by the National Natural Science Foundation of China under Grant No. 61501510 and No. 61301160, and Natural Science Foundation of Jiangsu Province under Grant No. BK20150717, and Jiangsu Planned Projects for Postdoctoral Research Funds.

Received: 26 November 2015 Accepted: 1 June 2016

Published online: 08 June 2016

\section{References}

1. Y Lien, C Chen, Y Lin, Toward ubiquitous massive accesses in 3GPP machine-to-machine communications. IEEE Commun. Mag. 49(4), 66-74 (2011)

2. G Ding, J Wang, Q Wu, Y Yao, F Song, TA Tsiftsis, Cellular-base-station assisted device-to-device communications in tv white space. IEEE J. Sel. Areas Commun. 34(1), 107-121 (2016)

3. M Song, CXin, Y Zhao, X Cheng, Dynamic spectrum access: from cognitive radio to network radio. IEEE Trans. Wireless Commun. 19(1), 23-29 (2012)

4. A Ghasemi, ES Sousa, Fundamental limits of spectrum-sharing in fading environments, vol. 6, (2007), pp. 649-658

5. L Musavian, S Aissa, Ergodic and outage capacities of spectrumsharing systems in fading channels. (IEEE GLOBECOM, Washington, America, 2007), pp. 3327-3331

6. X Chen, T Jing, Y Huo, W Li, X Cheng, T Chen. Achievable transmission capacity of cognitive radio network with cooperative relaying (IEEE CROWNCOM, Stockholm, Sweden, 2012), pp. 1-6

7. AT Hoang, YC Liang. A two-phase channel and power allocation scheme for cognitive radio networks. IEEE 17th International Symposium on Personal, Indoor and Mobile Radio Communications, (Helsinki, Finland, 2006), pp. 1-5

8. K Seong, M mohseni, JM Cioffi. Optimal resource allocation for OFDMA downlink systems (IEEE International Symposium on Information Theory, Seattle, America, 2006), pp. 1394-1398

9. G Li, H Liu, Downlink radio resource allocation for multi-cell OFDMA system. IEEE Trans. Wireless Commun. 5(12), 3451-3459 (2006)

10. H Zhu, Radio resource allocation for OFDMA systems in high speed environments. IEEE J. Sel. Areas Commun. 30(4), 748-759 (2012)

11. O El Ferkouss, W Ajib, Game theory based resource allocation for cognitive radio networks. IEEE Global Commun. Conf, 1174-1179 (2012)

12. G Zheng, KK Wong, B Ottersten, Robust cognitive beamforming with bounded channel uncertainties. IEEE Trans. Signal Process. 57(12), 4871-1881 (2009)

13. E Dall'Anese, SJ Kim, GB Giannakis, S Pupolin, Power control for cognitive radio networks under channel uncertainty. IEEE Trans. Wireless Commun. 10(10), 3541-3551 (2011)

14. IC Wong, BL Evans, Optimal resource allocation in the OFDMA downlink with imperfect channel knowledge. IEEE Trans. Wireless Commun. 57(1) 232-241 (2009)

15. R Aggarwal, M Assaad, CE Koksal, P Schniter, Joint scheduling and resource allocation in the OFDMA downlink: utility maximization under imperfect channel-state information. IEEE Trans. Signal Process. 59(11), 5589-5604 (2011) 
16. N Mokari, S Parsaeefard, H Saeedi, P Azmi, E Hossain, Secure robust ergodic uplink resource allocation in relay-assisted cognitive radio networks. IEEE Trans. Signal Process. 63(2), 291-304 (2015)

17. X Ma, F Li, J llow, Z Chen. Robust resource allocation for bidirectional decode-and-forward OFDM relaying systems with network coding and imperfect CSI. (IEEE Vehicular Technology Conference, Seoul, Korea, 2015), pp. 1-5

18. S Milana, A Fregolent, A Culla, Robust resource optimization for cooperative cognitive radio networks with imperfect CSI. IEEE Trans. Wireless Commun. 14(2), 907-920 (2015)

19. JB Wang, Q Su, J Wang, M Feng, Imperfect CSI-based joint resource allocation in multirelay OFDMA networks. IEEE Trans. Vehicular technology. 63(8), 3806-3817 (2014)

20. K Son, CJ Bang, C Song, KS Dan, Power allocation for OFDM-based cognitive radio systems under outage constraints. IEEE Intl. Conf. Commun. 29(16), 1-5 (2010)

21. Z Xue, L Shen, G-R Ding, Q-H Wu, Geolocation spectrum database assisted optimal power allocation: device-to-device communications in tv white space. KSII Trans. Internet Inf. Syst. 12(9), 4837-4855 (2015)

22. C-K Wen, S Jin, K-K Wong, J-C Chen, P Ting, Channel estimation for massive MIMO using gaussian-mixture bayesian learning. IEEE Trans. Wireless Commun. 14(3), 1356-1368 (2015)

23. J-W Huang, RA Berry, ML Honig, Distributed interference compensation for wireless networks. IEEE J. Sel. Areas Commun. 24(5), 1074-1084 (2006)

24. N Vucic, S Shi, M Schubert. DC programming approach for resource allocation in wireless networks, Modeling and Optimization in Mobile, Ad Hoc and Wireless Networks (WiOpt) 2010 Proceedings of the 8th International Symposium on, (Avignon, France, 2010), pp. 380-386

25. W Yu, R Lui, Dual methods for nonconvex spectrum optimization of multicarrier systems. IEEE Trans. Wireless Commun. 54(7), 1310-1322 (2006)

26. G-R Ding, Wu Q-H, J-L Wang, Sensing confidence level-based joint spectrum and power allocation in cognitive radio networks. Wireless Pers. Commun. 72(1), 283-298 (2013)

27. B Stephen, V Lieven, Convex optimization. (Cambridge University Press, Cambridge, 2009)

28. BV Gnedenko, AN Kolmogorov, Limit distributions for sums of independent random variables.reading. (Addison-Wesley, America, 1954)

29. H Hindi. A tutorial on convex optimization II: duality and interior point methods. (American Control Conference, Minneapolis, America, 2006), pp. 686-696

\section{Submit your manuscript to a SpringerOpen ${ }^{\circ}$ journal and benefit from:}

- Convenient online submission

- Rigorous peer review

- Immediate publication on acceptance

- Open access: articles freely available online

- High visibility within the field

- Retaining the copyright to your article

Submit your next manuscript at springeropen.com 